\title{
A Rare Case of Fungal Maxillary Sinusitis due to Paecilomyces lilacinus in an Immunocompetent Host, Presenting as a Subcutaneous Swelling
}

\author{
Harish S Permi, Sunil kumar Y, Vimal K Karnaker ${ }^{1}$, Kishan Prasad H L, \\ S Teerthanath, Satheesh Kumar Bhandary ${ }^{2}$
}

Departments of Pathology, ${ }^{1}$ Microbiology, ${ }^{2}$ ENT, K S Hegde Medical Academy of Nitte University, Deralakatte, Mangalore, India

Address for correspondence: Dr. Kishan Prasad HL, E-mail: dr_kishanpath@yahoo.com

\section{ABSTRACT}

Paecilomyces is a colonizing fungal species which usually causes keratitis, endocarditis, sinusitis, nephritis, fungemia, cutaneous, and subcutaneous infections in immunocompromised host. Very rarely, it causes similar infection in immunocompetent host without any risk factors. We report a case of maxillary sinusitis due to Paecilomyces lilacinus in a 65-year-old immunocompetent male, who presented with a subcutaneous swelling below the left eye. The lesion was excised by surgery and treated with itraconazole for 6 months based on culture and sensitivity. After 1 year of follow up, he is free of symptoms with no evidence of recurrence.

Keywords: Immunocompetent, itraconozole, maxillary sinusitis, Paecilomyces lilacinus

\section{INTRODUCTION}

\% ungal infections have become an important cause of morbidity and mortality in recent years, especially in immunocompromised patients. Paecilomyces species are saprophytic fungi found worldwide in soil and in decomposing vegetation. These belong to hyphomycetes class and are related to Penicillium and Aspergillus species. ${ }^{[1]}$ They usually occur as laboratory contaminants of sterile solutions and clinical specimens. ${ }^{[1]}$ In immunocompromised individuals, infection can occur virtually in any body site or organ system. ${ }^{[2]}$ Rarely disease has been reported in immunocompetent hosts without any identifiable risk factors. ${ }^{[3]}$ Five species in the genus Paecilomyces which cause infection include Paecilomyces variotii, Paecilomyces lilacinus, Paecilomyces marquandii, Paecilomyces viridis, and Paecilomyces javanicus. ${ }^{[4]}$ The most common species

\begin{tabular}{|l|l|}
\hline \multicolumn{3}{|c|}{ Access this article online } \\
\hline Quick Response Code: & Website: \\
\hline & \\
\hline
\end{tabular}

associated with human infections are $P$. lilacinus and $P$. variotii. ${ }^{[2]} \mathrm{It}$ is essential to identify the fungal infection in histopathology and definite species by culture for the specific treatment. We report a case of maxillary sinusitis by $P$. lilacinus in an immunocompetent host, who presented with left infraorbital swelling since 10 months. He was treated with surgery and oral itraconozole postoperatively. Disease responded well for the treatment. We emphasize the importance of histopathology, culture, and sensitivity for the identification of species in suspected cases of fungal sinusitis for accurate treatment and to prevent further complications.

\section{CASE REPORT}

A 65-year-old male presented with painless swelling below the left eye [Figure 1(a)] since 10 months. The swelling was insidious in onset and gradually increased in size. There was no history of trauma, nasal obstruction, nasal discharge, and visual disturbance. On examination, left infra orbital swelling was nontender and cystic ms $2 \times 2 \mathrm{~cm}$ with mild left maxillary sinus tenderness. Clinical diagnosis of epidermal cyst with maxillary sinusitis was made. 


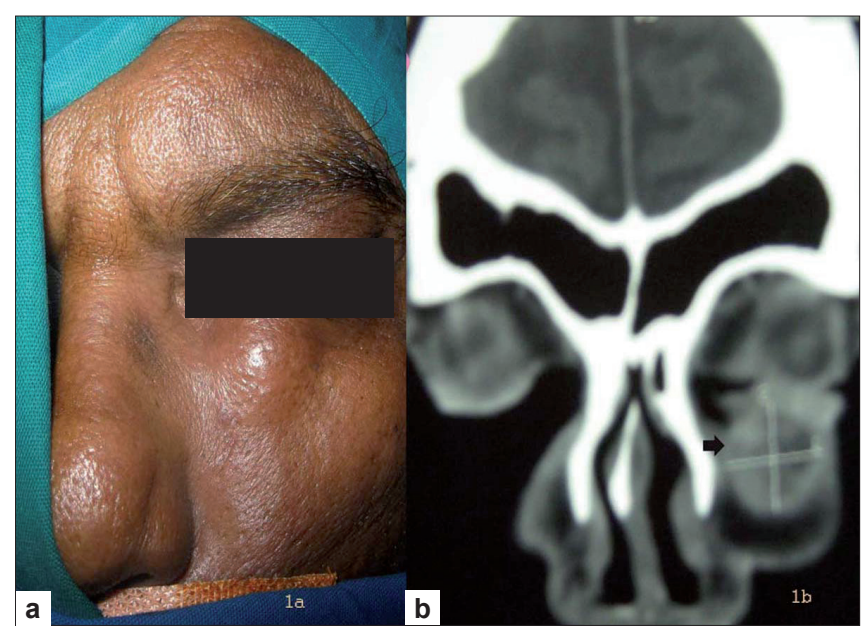

Figure 1: (a) Clinical image with left infraorbital swelling. (b) CT scan: paranasal sinuses showing well-defined rounded cystic lesion (shown with arrow) in the left infraorbital region, eroding the maxilla with focal thickening of left maxillary sinus mucosa

Hematological investigations revealed, hemoglobin - $13 \mathrm{gm} / \mathrm{dl}$, total leucocyte count - 9600 cells/cumm, platelet count of 1,89,000 cells/cumm and erythrocyte sedimentation rate of $18 \mathrm{~mm}$ at the end of $1 \mathrm{~h}$. Biochemical investigations showed random blood sugar - $98 \mathrm{mg} /$ $\mathrm{dl}$, blood urea - $38 \mathrm{mg} / \mathrm{dl}$, serum creatinine $-0.8 \mathrm{mg} / \mathrm{dl}$. Serology for HIV I and II by ELISA was negative. CT scan of paranasal sinuses showed well- defined rounded cystic lesion, $\mathrm{ms} 2.5 \times 1.8 \mathrm{~cm}$ in the subcutaneous tissue of the left infraorbital region, eroding the underlying bony orbital margin formed by the maxilla and focal thickening of left maxillary sinus mucosa [Figure 1(b)]. Excision of the left infraorbital cyst along with maxillary sinus clearance by Caldwell Luc's surgery was done.

Histopathological examination revealed fibrocollagenous tissue at the periphery, with central necrotic area surrounded by mixed inflammatory cells and refractile fungal hyphal elements. The Periodic acid-Schiff (PAS) and Gomori's methenamine silver stained sections showed fungal hyphal structures, which were broad, thin walled with infrequent septation and occasional branching [Figure 2(a)]. Based on these features, possibility of subcutaneous Entomophthoromycosis was considered and advised for fungal culture and sensitivity. On fungal culture, the colony was flat, powdery, velvety, and yellowish color. Reverse was white to pinkish in color. Microscopy showed elongated, tapering, phialides with a long slender tube, bent away from the axis of the conidiophores and appeared singly along the hyphae [Figure 2(b)]. The conidia were elliptical and arranged in long unbranched chains. Final diagnosis of left maxillary sinusitis due to $P$. lilacinus was made. Patient was treated with oral itraconozole $(200 \mathrm{mg})$ for 6 months, based on culture and sensitivity report. He was followed

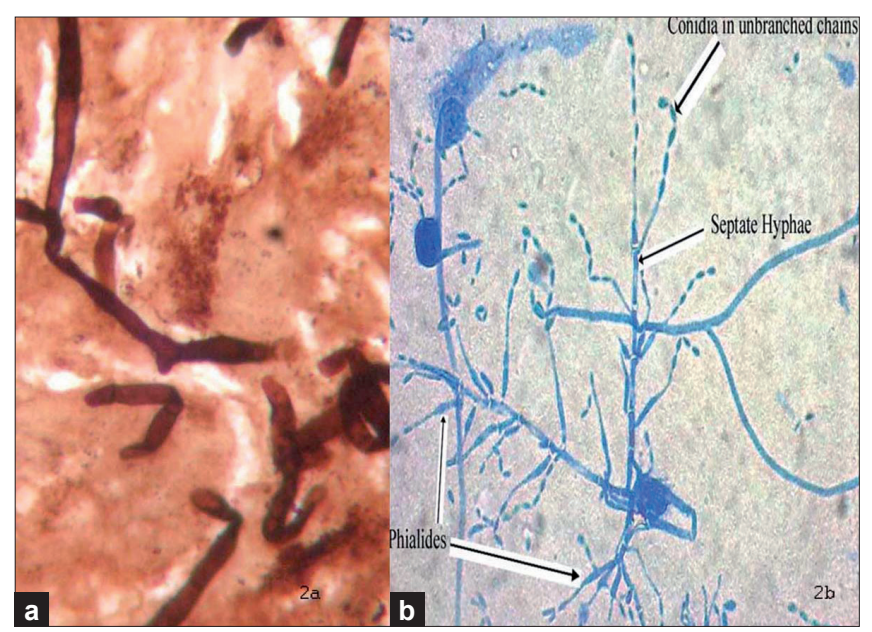

Figure 2: (a) Histopathology showing broad, thin walled, infrequent septate hyphae with occasional branching (GMS, $\times 400$ ). (b) Microscopy of the cultured $P$. lilacinus with characteristic finding (Lactophenol cotton blue, $400 x$ )

up with nasal endoscopy every month for a period of 1 year and was found to be free from signs and symptoms of recurrence.

\section{DISCUSSION}

Paecilomyces was first described by Bainier in 1907. ${ }^{[2]}$ It is a filamentous fungus, found in soil, decaying vegetation and is an infrequent cause of human disease. Most cases of disease caused by the genus Paecilomyces occur in patients who have compromised immune system, indwelling foreign devices, and intraocular lens implants.

It causes keratitis, endocarditis, sinusitis, nephritis, fungemia, pulmonary, cutaneous, and subcutaneous infections. ${ }^{[3]}$ The earliest report of infection from Paecilomyces was a case of empyema, caused by P. lilacinus in $1972 .{ }^{[5]}$ In recent years, there have been increasing reports of this infection in patients without any predisposing factors, producing vaginitis, sinusitis, and pleural effusion. ${ }^{[6]}$ Although definitive identification of these fungi requires culture, they often can be identified provisionally on tissue sections. The histologic features include hyaline septate hyphae and characteristic reproductive structures such as phialides and phialoconidia. These morphologic characteristics, although familiar to mycologists, are easily overlooked by histopathologists; as a result P. lilacinus are frequently misidentified in tissue sections as Aspergillus or Candida species. ${ }^{[4]}$ The fungal hyphae are broad, infrequent branching, twisted, knotted eosinophilic appearance on Haematoxylin and Eosin stain. On PAS stain, they appear deep red with large, bulbous structures. ${ }^{[7]}$ Our case also had similar histological features. 
On Saborauds Dextrose Agar media, P. lilacinus grow rapidly and mature within 3 days. The colonies are flat, powdery, or velvety in texture. The color is initially white and becomes yellow, yellow-green, or yellowbrown. The reverse is dirty white, buff or brown. Septate hyaline hyphae, conidiophores, phialides, conidia, and chlamydospores are observed. Conidiophores are often branched and carry the phialides at their tips. The phialides are swollen at their bases and taper toward their apices. Conidia are unicellular and form long chains. Chlamydospores are occasionally present. ${ }^{[8]}$ Our case also showed the similar features.

P. lilacinus is emerging as important pathogen, since it is resistant to many antifungal drugs including amphotericin B. In contrast to the other species belonging to this genus, P. lilacinus generally shows a poor response to conventional antifungal drugs. Therefore, correct identification of the species is mandatory for appropriate treatment of the disease. Many therapeutic regimens have been used to treat paecilomycosis, but with a high failure rates. ${ }^{[1,9]}$ In these cases, surgery must be combined with antifungal agents for complete cure. The older antifungal drugs such as amphotericin B, flucytosine, fluconazole, miconazole, and itraconazole have been used in many cases of cutaneous, sub-cutaneous, and ocular infections. ${ }^{[9]}$ Maria et al, reported P. lilacinus sinusitis in a bone marrow transplant recipient and treated with amphotericin B with good response. ${ }^{[1]}$ The azoles are considered a better choice than amphotericin B against this particular species of Paecilomyces. ${ }^{[10]}$ Gucalp et al, described a case of sinusitis by P.lilacinus resistant in vitro to amphotericin $\mathrm{B}$, but susceptible to itraconazole. ${ }^{[1]}$ Gutierrez- Rodero et al, ${ }^{[10]}$ reported cutaneous infection by $P$. lilacinus in a healthy patient, where prolonged antifungal therapy with itraconozole led to resolution of skin lesion. Although there is limited clinical experience in the use of voriconazole, this drug seems to be the most effective agent for the treatment of these fungal infections. ${ }^{[0]}$ However in our case, based on culture and sensitivity analysis, treatment with itraconazole had good clinical response.
We conclude that definite fungal species identification is necessary for specific treatment with antifungal agents, for complete cure and to prevent further recurrence.

\section{ACKNOWLEDGMENTS}

We are thankful to Dr. Vadisha Bhat, Assistant Professor in ENT, Dr. J H Makannavar, Senior Professor of Pathology, Dr. Jayaprakash Shetty K, Professor and Head, Department of Pathology for their valuable help and suggestions.

\section{REFERENCES}

1. Maria CR, David RS, Kenneth M. Paecilomyces lilacinus sinusitis in a Bone marrow transplant recipient: Case report and review of the literature. Infect Dis Clin Pract 1996;5:311-4.

2. Varkey JB, Perfect JR. Rare and Emerging Fungal Pulmonary Infections. Semin Respir Crit Care Med 2008;29:121-31.

3. Carey J, D'Amico R, Sutton D, Rinaldi MG. Paecilomyces lilacinus vaginitis in an Immunocompetent Patient. Emerg Infect Dis 2003;9:1155-8.

4. Liu K, Howell DN, Perfect JR, Schell WA. Morphologic criteria for the preliminary identification of Fusarium, Paecilomyces and Acremonium species by histopathology. Am J Clin Pathol 1998;109:45-54.

5. Fenech FF, Mallia CP. Pleural effusion caused by Penicillium lilacinum. Br J Dis Chest 1972; 66:284-90.

6. Gutierrez F, Masia M, Ramos J, Elia M, Mellado E, uenca-Estrella M. Pulmonary mycetoma caused by an atypical isolate of paecilomyces species in an immunocompetent individual: Case report and literature review of paecilomyces lung infections. Eur J Clin Microbiol Infect Dis 2005;24:607-11.

7. Rockhill RC, Klein MD. Paecilomyces lilacinus as the cause of Chronic Maxillary Sinusitis. J Clin Microbiol 1980;11:737-9.

8. Davise H, Larone MT. Medically important fungi. A guide to identification. $4^{\text {th }}$ ed. United States: American society for Microbiology; 2002. p. 270-4.

9. Pastor FJ, Guarro J. Clinical manifestations, treatment and outcome of Paecilomyces lilacinus infections. Clin Microbiol Infect 2006;12:948-60.

10. Gutiérrez-Rodero F, Moragón M, Ortiz de la Tabla V, Mayol MJ, Martín C. Cutaneous Hyalohyphomycosis caused by Paecilomyces lilacinus in an immunocompetent host successfully treated with itraconozole: Case report and review. Eur J Clin Microbiol Infect Dis 1999;18:814-8.

11. Gucalp R, Carlisle P, Gialanella P, Mistudo S, Mckitrick J, Dutcher J. Paecilomyces sinusitis in an immunocompromised adult patient: Case report and review. Clin Infect Dis 1996;23:391-3.

How to cite this article: Permi HS, kumar SY, Karnaker VK, Prasad KH, Teerthanath S, Bhandary SK. A rare case of fungal maxillary sinusitis due to Paecilomyces lilacinus in an immunocompetent host, presenting as a subcutaneous swelling. J Lab Physicians 2011;3:46-8.

Source of Support: Nil. Conflict of Interest: None declared. 